\title{
High-resolution gamma-ray detection using phonon-mediated detectors
}

\author{
B. Cornell ${ }^{a}$, D.C. Moore ${ }^{a}$, S.R. Golwala ${ }^{a}$, B. Bumble ${ }^{b}$, P.K. Day ${ }^{b}$, H.G. LeDuc ${ }^{b}$, and \\ J. Zmuidzinas ${ }^{a}$ \\ ${ }^{a}$ California Institute of Technology, 1200 E. California Blvd, Pasadena, CA, USA, 91125; \\ ${ }^{b}$ Jet Propulsion Laboratory, 4800 Oak Grove, Pasadena, CA, USA, 91109
}

\begin{abstract}
We are presenting the results of our ongoing efforts to develop a new type of focal plane detector for the 10 to 100 $\mathrm{keV}$ band with an energy resolution of $0.1 \%$. The device will measure energy and position by using microwave kinetic inductance detectors (MKIDs) to sense athermal phonons created by photon absorption in a dielectric substrate. We have fabricated a proof-of-concept detector of size $2 \mathrm{~cm} \times 2 \mathrm{~cm} \times 1 \mathrm{~mm}$ on silicon, which has demonstrated a baseline energy resolution of $=0.38 \mathrm{keV}$ and $=0.55 \mathrm{keV}$ at $30 \mathrm{keV}$.
\end{abstract}

Keywords: Kinetic inductance detectors, Phonon, Gamma ray, X-ray

\section{INTRODUCTION}

The recent breakthroughs in hard X-ray/soft $\gamma$-ray optics have finally opened up the 10-100 keV band to detailed astronomical observation. ${ }^{1}$ At these energies, where particle interaction backgrounds are of particular concern, large concentration factors $(>1000)$ are enormously important for improving sensitivity. The newest generation of X-ray focusing telescopes, such as The Nuclear Spectroscopic Telescope Array (NuSTAR) and the proposed New Hard X-ray Mission (NHXM) extend this kind of light collection power to the decade above $10 \mathrm{keV}$. In this region, the 67.9 and $78.4 \mathrm{keV} \gamma$-ray lines arising from the decay of ${ }^{44} \mathrm{Ti}$ are of particular interest. ${ }^{2,3} \mathrm{In}$ supernova remnants, this isotope forms the boundary between the ejected matter that falls back to form the daughter neutron star or black hole and that which escapes. The location and dynamics of this so called "mass cut" depend strongly on the explosion mechanism of the supernova. The underlying physics of this process is not well understood, and is a compelling area of study in its own right, but supernovae also substantially affect the evolution of galaxies, the intergalactic, and interstellar medium. Being the progenitor and the distribution mechanism for the heavy elements, supernovae are vital to a deeper understanding of our own origins.

Typical velocities of the material at the mass cut would be on order of a few hundred $\mathrm{km} / \mathrm{s}$. Meaningful velocity measurements would require sensitivity to the resulting $\sim 0.1 \%$ Doppler shift. The current state-of-the art CdZnTe detectors, such as those used in NuSTAR, have a FWHM spectral resolution of around $1 \mathrm{keV}$ at 70 $\mathrm{keV}$, or $\sim 1 \%$, over an area of a few square $\mathrm{cm}$. We are developing a phonon based technology to recover this order of magnitude in sensitivity.

\section{PHONON MEDIATED KINETIC INDUCTANCE DETECTORS}

\subsection{OVERVIEW}

The energy required to create an electron hole pair in a typical semiconductor is on order of a few $\mathrm{eV}(\sim 4.5 \mathrm{eV}$ for $\mathrm{CdZnTe}$ ). This would limit any ionization-based measurement of the 10 to $100 \mathrm{KeV}$ decade of interest to a resolution of $\sigma \approx 0.03 \%$ simply from Fano corrected Poisson fluctuations. ${ }^{4}$ This fundamental barrier can be overcome, while still utilizing the development and fabrication benefits provided by semiconductor technology, by simply reading out a quasiparticle that is produced in more abundance. A simple solution would be to read out the phonon signal in a cryogenic semiconductor crystal using a pair-breaking thin film detector such as a Microwave Kinetic Inductance Detector (MKID). ${ }^{5}$ A phonon-mediated MKID-based detector would consist of

Further author information: (Send correspondence to B. Cornell)

B. Cornell: E-mail: cornell@caltech.edu, Telephone: 15302120121

High Energy, Optical, and Infrared Detectors for Astronomy V, edited by Andrew D. Holland, James W. Beletic, Proc. of SPIE Vol. 8453, 84532N · C 2012 SPIE · CCC code: 0277-786X/12/\$18 · doi: 10.1117/12.926829 


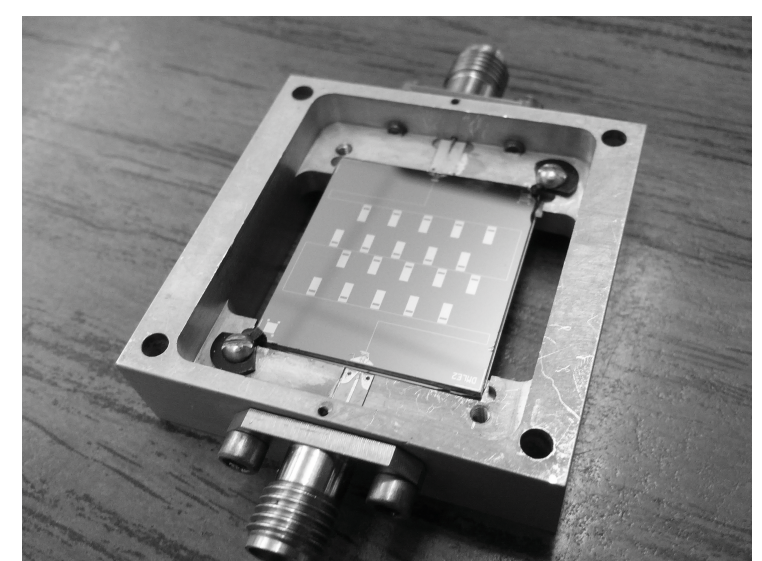

Figure 1: Our $2 \mathrm{~cm} \times 2 \mathrm{~cm} \times 1 \mathrm{~mm}$ test device mounted in its holder. (a)

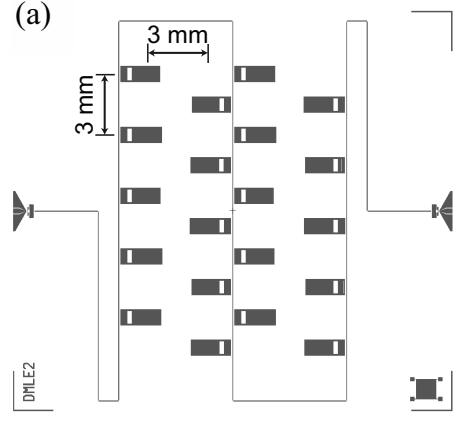

(b)

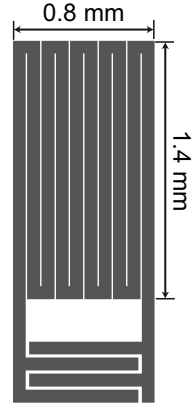

Figure 2: (a) Array layout. The resonators are arranged in an offset grid with $3 \mathrm{~mm}$ spacing between the neighboring rows and columns and are coupled to a coplanar strip feedline. (b) Resonator geometry. Each resonator consists of a meandered inductor connected to an interdigitated capacitor, with total inductor area $\approx 1 \mathrm{~mm}^{2}$.

hundreds of MKIDs patterned on both faces of a germanium or silicon substrate. Particle interactions in the substrate produce phonons at the Debye frequency, which rapidly downconvert until their mean free path is comparable to the size of the substrate $(\nu \approx 0.5 \mathrm{THz}){ }^{6}$ These "ballistic" phonons can reach the MKIDs on the surface and break Cooper pairs, leading to a shift in the frequency and dissipation in the resonator.

To calculate the energy resolution of a phonon-based MKID detector we assumed that the surface of the substrate is uniformly illuminated by the phonons which convert to quasiparticles in the inductive portion of the MKID with an overall efficiency of $\eta_{p h}$. Under this assumption, the phonon lifetime, $\tau_{p h}$, depends only on the fraction of the substrate surface area that is covered with MKIDs where $\eta_{f i l l}=A_{M K I D s} / A_{\text {sub }}$. To resolve the rising edge of the phonon signal requires resonator response times of approximately $\tau_{\text {res }} \approx 1 \mu \mathrm{s}$. For readout frequencies around $f_{0} \approx 3 \mathrm{GHz}$ this requires a quality factor, $Q$, of $Q=\tau_{r e s} \pi f_{0} \leq 10^{4}$. Typically we divide the quality factor into two pieces: the coupling quality factor, $Q_{c}$, is due to the coupling of the MKID to the readout system (in our case a CPS feedline), and the internal quality factor, $Q_{i}$, which is due to all other loss mechanisms (e.g. dissipation from quasiparticles) where $Q^{-1}=Q_{c}^{-1}+Q_{i}^{-1}$. For our devices, $Q_{i}>10^{6}$, and to insure that $Q \leq 10^{4}$, the energy loss of the MKID should be dominated by coupling to the feedline or $Q_{c} \ll Q_{i}$. We also assume that the population of thermal quasiparticles is negligible and that the steady-state quasiparticle number is due to the readout power, as in $N_{q p}=\eta_{\text {read }} P_{\text {read }} \tau_{q p} / \Delta$ where $P_{\text {read }}$ is the readout power, $\Delta$ is the superconducting gap and $\eta_{\text {read }}$ is an efficiency correction which is conservatively unity. Last, we assume the our primary HEMPT amplification stage, with noise temperature $T_{N}$, dominates our readout noise. Given this set of assumptions the energy resolution from a phonon-mediated MKID detector is ${ }^{7}$

$$
\sigma_{E}=\frac{\Delta}{\eta_{p h} \beta(\omega, T)} \sqrt{\frac{\eta_{\text {read }}}{\alpha p_{t}} \frac{A_{s u b} k_{b} T_{N}}{2 \pi f_{0}} \frac{N_{0} \lambda_{p b}}{\tau_{q p} S_{1}(\omega, T)}}
$$

where $p_{t}$ is the probability for a phonon to be transmitted from the substrate to the MKID, $\alpha$ is the fraction of the total inductance of the resonators due to kinetic inductance, and $\lambda_{p b}$ is the characteristic pair-breaking length in the MKID. The ratio of the frequency to dissipation response is defined as $\beta=S_{2} / S_{1}$, where $S_{1}(\omega, T)$ and $S_{2}(\omega, T)$ are dimensionless factors of order unity given by Mattis-Bardeen theory. ${ }^{8,9}$

\section{SQUARE CENTIMETER TEST DEVICE}

\subsection{DESIGN}

As a proof of principle of this detection technique, and to verify the energy resolution in Eq. 1 we designed and fabricated a prototype device. It consists of a single $25 \mathrm{~nm}$ thick Al film photolithographically patterned 


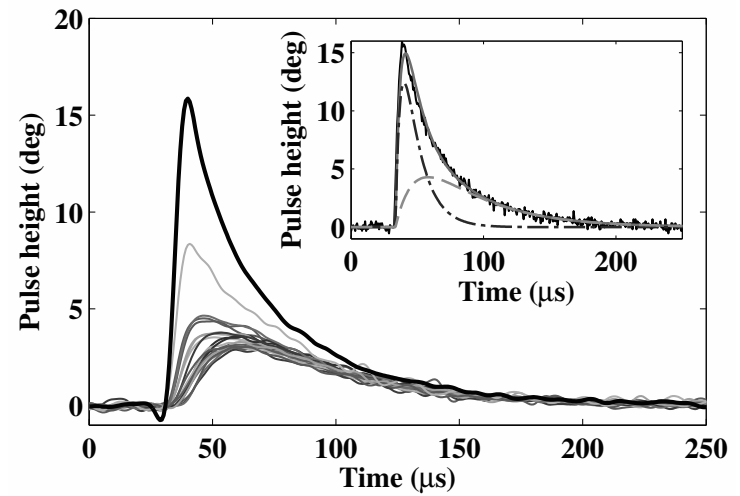

Figure 3: Example phase response of a $200 \mathrm{keV}$ cosmic ray event. The pulses have all been normalized against their respective MKID's relative responsively, and an anti-aliasing filter has been applied. The resonator closest to the interaction site (thick black pulse) has both the greatest energy partition as well as the most prompt response. (inset) This is used to construct a two template optimal filter fit to the data (sold fit). This contains a prompt component (dotted-dashed) that reflects the quasiparticle lifetime in the MKID as well as a slower component (dashed) representing the phonon lifetime in the substrate.

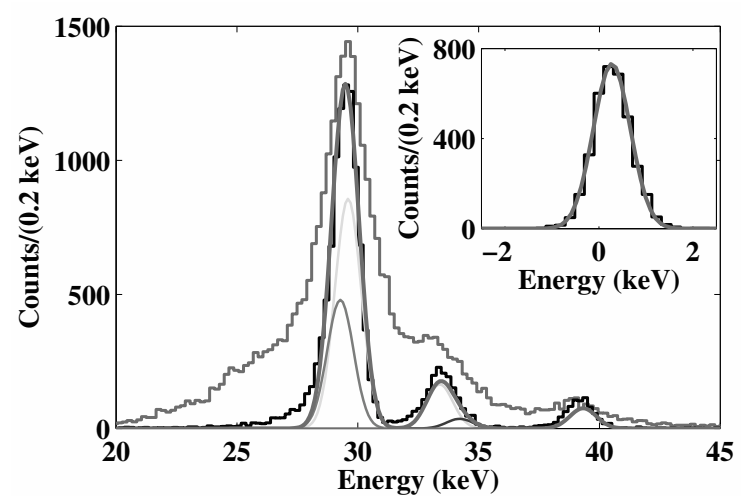

Figure 4: Observed spectrum when the substrate face opposite the resonators is illuminated with $\mathrm{x}$-rays from an ${ }^{129}$ I source. The light histogram shows the reconstructed energy for all events prior to correcting for position-dependent smearing of the response; the dark histogram is after. The best fit resolution at $30 \mathrm{keV}$ is $\sigma_{E}=0.55 \mathrm{keV}$. (inset) Fit to the reconstructed energy spectrum for randomly triggered noise traces. The inferred baseline resolution is $\sigma_{E}=0.38 \mathrm{keV}$.

on a $1 \mathrm{~mm}$ Si substrate. The resonators were designed with an inductive area $A_{\text {ind }}=1 \mathrm{~mm}^{2}$, and to keep the active metal coverage at $5 \%$, they were fashioned into an array of 20 MKIDs as seen in Fig. 2. The resonator design consists of a lumped-element geometry, ${ }^{10}$ with a 70 micron wide meandered inductor connected to an interdigitated capacitor with 20 micron spacing between fingers. The resonant frequencies were evenly spaced by $10 \mathrm{MHz}$ so that the entire array fit within $200 \mathrm{MHz}$ of bandwidth around $4.8 \mathrm{GHz}$. An ${ }^{129} \mathrm{I}$ source was mounted to illuminate the substrate face opposite the resonators, and the enclosure was cooled to $50 \mathrm{mK}$ in a dilution refrigerator.

\subsection{READOUT AND POSITION CORRECTION}

The open source readout system utilizing FPGA-based signal processing hardware developed by CASPER ${ }^{11}$ was used to excite the resonators and read out the array. Custom firmware was developed for this system implementing a 20-channel digital downconverter (DDC) to isolate each frequency channel. The system provides the amplitude and phase of the signal transmitted past each resonator at $1.3 \mathrm{MHz}$ per channel, with onboard buffering and triggering. The total system bandwidth is $340 \mathrm{MHz}$, easily encompassing the $200 \mathrm{Mhz}$ bandwidth of the array. An example of the phase response for each of the 20 resonators following a $200 \mathrm{keV}$ interaction in the substrate from a cosmic-ray can be seen in Fig. 3. The location of the interaction can be reconstructed from the relative fraction of energy partitioned into each of the 20 MKIDs. ${ }^{12,13}$ This allows for a local energy recalibration to be preformed as seen in Fig. 4. The best-fit resolution after the position dependent correction is $\sigma=0.55 \mathrm{keV}$ at $30 \mathrm{keV}$. This is almost a factor of 2 better than the uncorrected resolution and brings us within $40 \%$ of the baseline resolution of $\sigma=0.38 \mathrm{keV}$. This is consistent with our expectations from Eq. 1 .

\section{THREE INCH DEVICE DEVELOPMENT}

\subsection{OVERVIEW}

The position correction analysis of our square centimeter devices has allowed us to essentially recover our baseline energy resolution of a few hundred $\mathrm{eV}$. Our next device is an early focal-plane prototype; a substantial change from our current proof-of-concept detectors. To fully stop $100 \mathrm{keV} \gamma$ 's in Ge requires a substrate thickness 


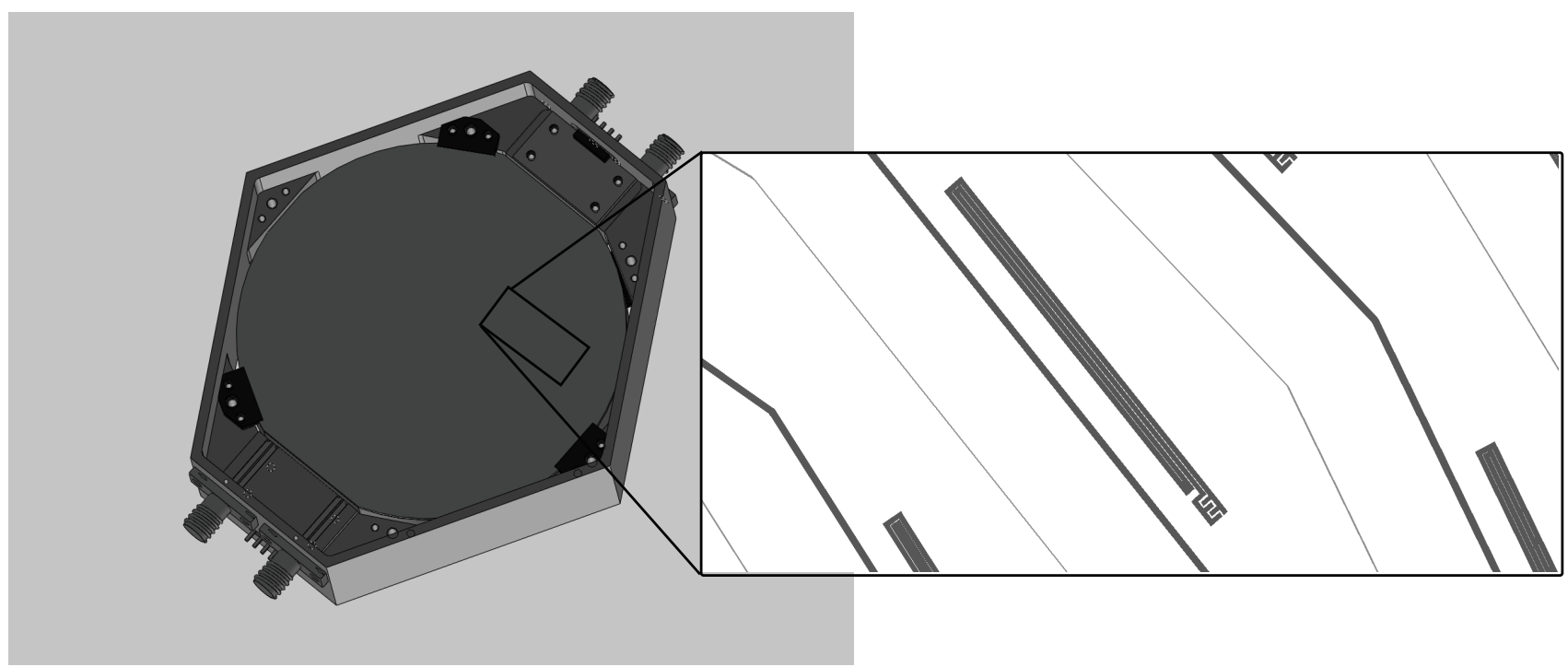

Figure 5: A mock up of the newly designed three inch device mounted in its holder. It will be patterned with 250 MKIDS on each surface, which will be read out separately. The mount allows for the readout of both faces of the device, each with its own co-ax to room temperature. (insert) A detail of the newly designed mask.

of $1 \mathrm{~cm}$. Much work has been done on phonon readout from thick Ge and Si substrates for use in rare event searches, such as direct dark matter detection, ${ }^{14}$ neutrinoless double-beta decay ${ }^{15}$ and coherent neutrino-nucleus scattering. ${ }^{16}$ While energy threshold typically plays a more important role than energy resolution in designing a detector for low statics physics, they provided a useful starting point in thinking about metal coverage, and expected efficiency. ${ }^{14}$ It was these considerations, as well as the capabilities of our fabrication and cryogenic facilities which led us to settle on a three inch wafer for our next device as seen in Fig. 5. The pictured mount has already been fabricated, and is designed to minimise phonon loss from the crystal to the mount. This was thought to be one of the major phonon loss mechanisms in our previous devices and the much larger mass to contact area ratio afforded by the new substrate/mount combination should help increase our phonon collection efficiency.

\subsection{RESONATOR AND MASK DESIGN}

The size of the new MKIDs was set by our readout electronics and our desire to keep our active metal coverage at the few percent level. Our current FPGA channelizer and GHz mixer have a lower operating frequency limit of $3 \mathrm{GHz}$, which sets an effective upper limit on the size of our resonators. We are also currently limited to multiplexing 250 MKIDs per FPGA board, which together with our coverage requirement, sets a lower limit. Resonators that are $\sim 1 \mathrm{~mm}^{2}$ allow for 250 MKIDs to be designed for the 3 to $3.5 \mathrm{GHz}$ band while maintaining a $1.4 \%$ coverage. This device will utilize a single $25 \mathrm{~nm} \mathrm{Al} \mathrm{film,} \mathrm{and} \mathrm{although} \mathrm{it} \mathrm{is} \mathrm{optimized} \mathrm{for} \mathrm{a} \mathrm{Ge} \mathrm{substrate,}$ it is designed to be functional on $\mathrm{Si}$ as well.

In addition to the phonon based readout, charge lines have been added which may be used to bias the substrate and readout a complementary ionization signal. This would allow us to make an absolute energy calibration of our device, as well as a fast feducialization technique that does not require a full position reconstruction. The addition of these charge lines, which are interleaved with the MKID feedline, sets a limit on the distance the MKID can protrude from the feedline to $\sim 500$ microns. The final consideration we made when designing our new resonators was to ensure that no edge formed a dipole antenna in the far field, to minimize cross resonator coupling. With these constraints in mind, we settled on a simple "fold over" form factor for our meandered inductor as seen in Fig. 6. The MKID is $200 \times 4000$ microns. The inductive section is 45 microns with a 7 micron gap; the interdigitated capacitor fingers are 40 microns with a 20 micron gap. These resonators may be tuned to different resonant frequencies by changing the length of the inductive section. Using SONNET's high frequency electromagnetic simulation software we were able to simulate the frequency response of our design and 
Figure 6: Our new MKID design for our three inch device. It is $200 \times 4000$ microns. The "fold over" design of the inductive section ensures that countervailing currents prevent the resonator from dipole radiating.

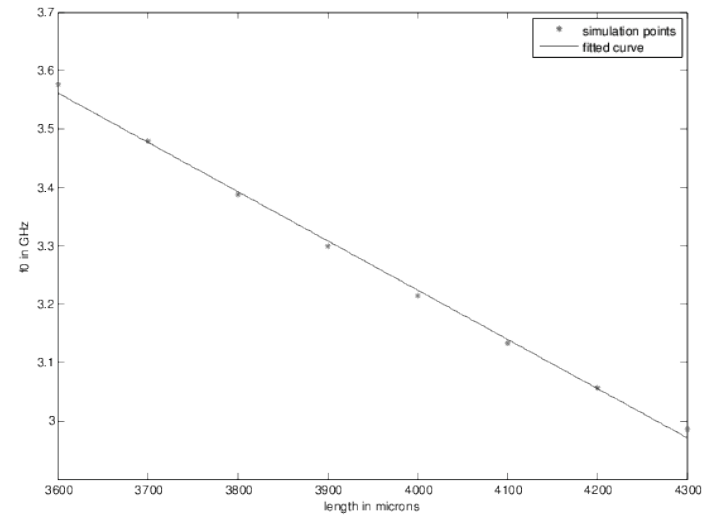

Figure 7: Resonant frequency, GHz, vs. MKID length, microns, from simulation of a single isolated resonator above a straight section of feedline. Assuming there is no unwanted coupling to other features of the mask, this lets us control the frequency encoding of our MKIDs simply by changing their length.

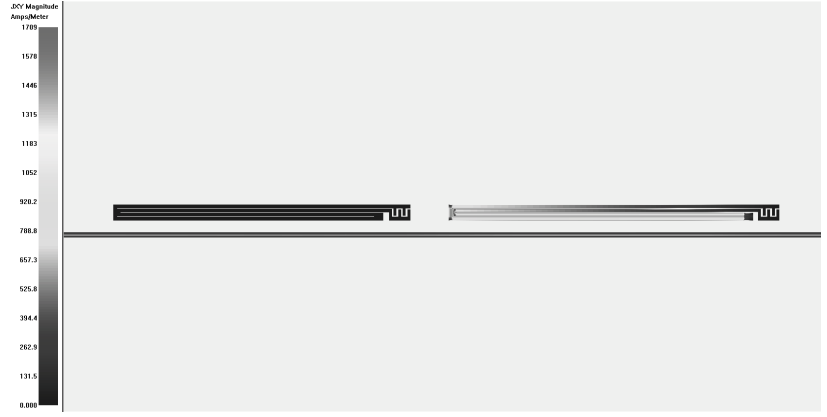

(a) Current response for an azimuthal coupling simulation. Even for very small separations, there is very little current in the off-resonant MKID. There is also no appreciable shift in resonant frequency for either resonator in this configuration.

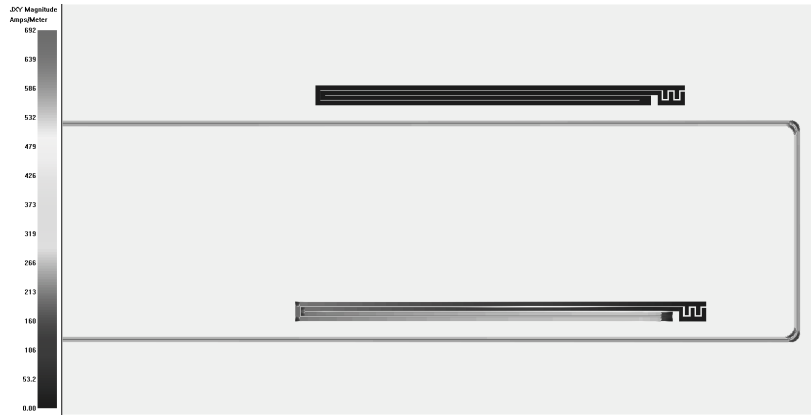

(b) Current response for a radial coupling simulation. As with the azimuthal simulation, there is no cross-coupling, however, the lower MKID does experience a shift in its resonant frequency from coupling to the vertical feedline segment.

Figure 8

as seen in Fig. 6, it is closely linear. We then used this fit to design 250 resonators with resonant frequencies evenly spaced in the $500 \mathrm{MHz}$ between 3 and $3.5 \mathrm{GHz}$.

\subsection{COUPLING SIMULATION AND CORRECTIONS}

To ensure that our resonators respond at their design frequency, we conducted a series of coupling simulations to ensure there was no cross-talk between nearest neighbor pairs of resonators. The physics of the phenomena for a specific MKID geometry is discussed in detail elsewhere. ${ }^{17}$ Due to computing resource constraints, we approximated our inter MKID coupling with basic geometries, radial and azimuthal. These approximations allowed us to ensure that we could design non-interacting resonators wile keeping an efficient Manhattan geometry for simulation purposes. As can be seen in Fig. 8, for MKIDs reasonably spaced in frequency space, there is no cross-coupling for either the radial or azimuthal pair. However, during the radial coupling simulations, we did discover unwanted coupling from the lower MKID to the vertical feedline segment. The difference between the actual length of the MKID and the expected length of the MKID based on the initial free resonator simulations 


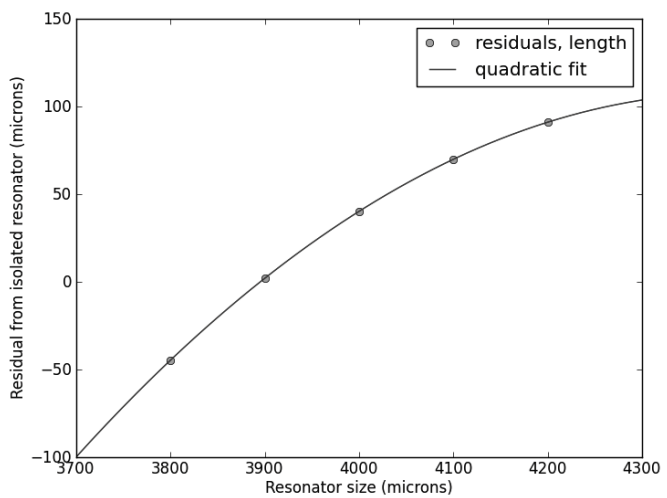

Figure 9: Residuals of an MKID's length at a constant resonant frequency with and without vertical feedline coupling vs. resonator length. They are fit to a quadratic, which can be used to correct the frequency shift due to coupling to a vertical segment of feedline.

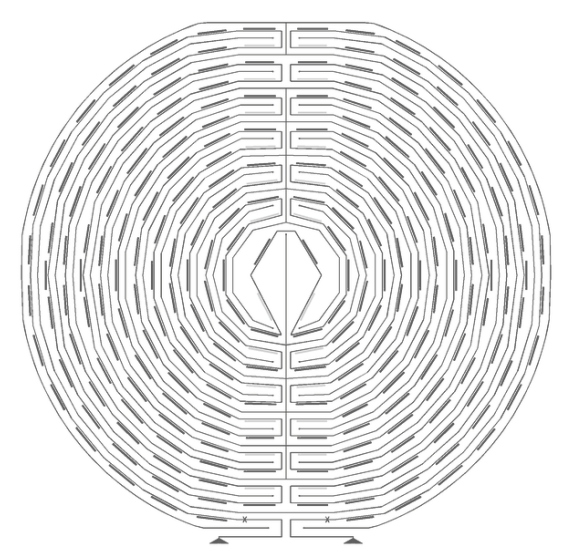

Figure 10: The full three inch mask design. The light grey resonators on either side of the vertical axis have had the quadratic length correction applied.

for this geometry is quadratic as a function of resonator length as seen in Fig. 9. We applied this correction to the affected resonators as seen if Fig. 10.

The final frequency encoding of the resonators needs to ensure that nearby MKIDs are sufficiently far in frequency space as to not interfere with one another. Our previous device used a random placement, however this proved to add unnecessary compilation to analysis, especially if two or more MKIDs were change the order of their resonant frequencies. For the three inch mask we divided our resonators into six equal subdomains, and alternate between domains as the MKIDs are placed along the feedline, azimuthally as seen in Fig. 11. This device is scheduled to be fabricated on a thin Si substrate in the coming weeks, so that the mask design may be $\mathrm{RF}$ tested prior to the manufacture of a thick Ge device.

\section{ACKNOWLEDGMENTS}

Our research was carried out primarily at the Jet Propulsion Laboratory (JPL) and the California Institute for Technology, under contract from NASA. JPL's Microdevices Laboratory fabricated the devices used in this work. We would like to acknowledge the generous support of the Gordon and Betty Moore Foundation. Our work benefited greatly from the expertise of, as well as software developed by, the CDMS/SuperCDMS collaboration for the development of phonon-mediated particle detectors. We would also like to thank NASA's Space Technology Research Fellowship program for its ongoing support.

\section{REFERENCES}

[1] Harrison, F. A., Boggs, S., Christensen, F., Craig, W., Hailey, C., Stern, D., Zhang, W., Angelini, L., An, H., Bhalereo, V., Brejnholt, N., Cominsky, L., Cook, W. R., Doll, M., Giommi, P., Grefenstette, B., Hornstrup, A., Kaspi, V., Kim, Y., Kitaguchi, T., Koglin, J., Liebe, C. C., Madejski, G., Kruse Madsen, K., Mao, P., Meier, D., Miyasaka, H., Mori, K., Perri, M., Pivovaroff, M., Puccetti, S., Rana, V., and Zoglauer, A., "The Nuclear Spectroscopic Telescope Array (NuSTAR)," in [Society of Photo-Optical Instrumentation Engineers (SPIE) Conference Series], Presented at the Society of Photo-Optical Instrumentation Engineers (SPIE) Conference $\mathbf{7 7 3 2}$ (2010).

[2] Martin, P., Knödlseder, J., Vink, J., Decourchelle, A., and Renaud, M., "Constraints on the kinematics of the ${ }^{44} \mathrm{Ti}$ ejecta of Cassiopeia A from INTEGRAL/SPI," Astronomy and Astrophysics 502, 131-137 (July 2009). 


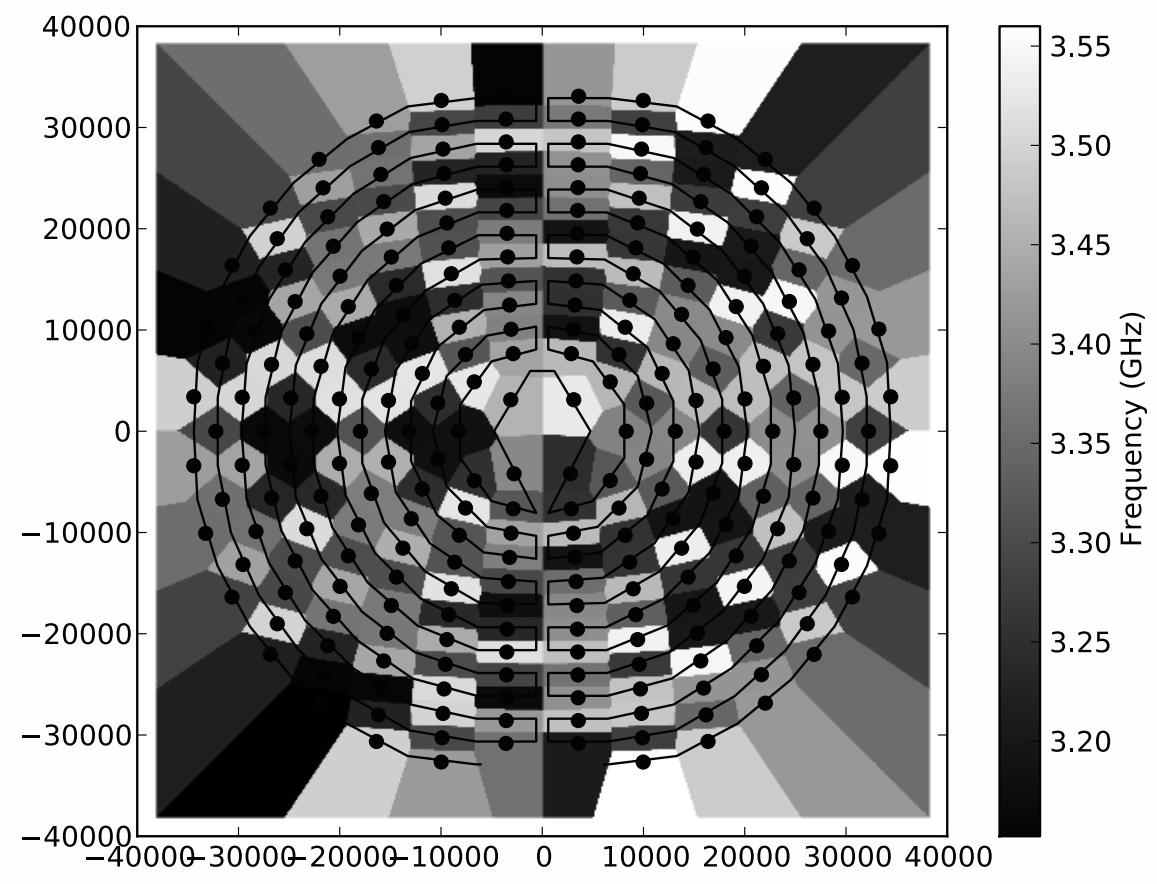

Figure 11: The frequency encoding for our three inch mask design. The $x$ and $y$ axes represent the position from the center of the wafer (in microns). The feedline (black line) and resonator positions (black dots) are also indicated

[3] Diehl, R. and Timmes, F. X., "Gamma-Ray Line Emission from Radioactive Isotopes in Stars and Galaxies," The Publications of the Astronomical Society of the Pacific 110, 637-659 (June 1998).

[4] Beringer, J. et al. (Particle Data Group) Phys. Rev. D86, 010001/1-70 (2012).

[5] Day, P. K., LeDuc, H. G., Mazin, B. A., Vayonakis, A., and Zmuidzinas, J., "A broadband superconducting detector suitable for use in large arrays," Nature 425, 817-821 (Oct. 2003).

[6] Kazakovtsev, D. V. and Levinson, Y. B., "Phonon diffusion with frequency down-conversion," Physica Status Solidi B Basic Research 96, 117-127 (Nov. 1979).

[7] Moore, D. C., Golwala, S., Bumble, B., Cornell, B., Mazin, B. A., Gao, J., Day, P. K., LeDuc, H. G., and Zmuidzinas, J., "Phonon Mediated Microwave Kinetic Inductance Detectors," Journal of Low Temperature Physics 167, 329-334 (May 2012).

[8] Mattis, D. C. and Bardeen, J., "Theory of the Anomalous Skin Effect in Normal and Superconducting Metals," Physical Review 111, 412-417 (July 1958).

[9] Gao, J., The Physics of Superconducting Microwave Resonators, PhD thesis, California Institute of Technology (2008).

[10] Doyle, S., Mauskopf, P., Naylon, J., Porch, A., and Duncombe, C., "Lumped Element Kinetic Inductance Detectors," Journal of Low Temperature Physics 151, 530-536 (Apr. 2008).

[11] Parsons, A., Backer, D., Siemion, A., Chen, H., Werthimer, D., Droz, P., Filiba, T., Manley, J., McMahon, P., Parsa, A., MacMahon, D., and Wright, M., "A Scalable Correlator Architecture Based on Modular FPGA Hardware, Reuseable Gateware, and Data Packetization," Publications of the Astronomical Society of the Pacific 120, 1207-1221 (Nov. 2008).

[12] CDMS II Collaboration, Ahmed, Z., Akerib, D. S., Arrenberg, S., Bailey, C. N., Balakishiyeva, D., Baudis, L., Bauer, D. A., Brink, P. L., Bruch, T., Bunker, R., Cabrera, B., Caldwell, D. O., Cooley, J., Cushman, P., Daal, M., DeJongh, F., Dragowsky, M. R., Duong, L., Fallows, S., Figueroa-Feliciano, E., Filippini, J., 
Fritts, M., Golwala, S. R., Grant, D. R., Hall, J., Hennings-Yeomans, R., Hertel, S. A., Holmgren, D., Hsu, L., Huber, M. E., Kamaev, O., Kiveni, M., Kos, M., Leman, S. W., Mahapatra, R., Mandic, V., McCarthy, K. A., Mirabolfathi, N., Moore, D., Nelson, H., Ogburn, R. W., Phipps, A., Pyle, M., Qiu, X., Ramberg, E., Rau, W., Reisetter, A., Saab, T., Sadoulet, B., Sander, J., Schnee, R. W., Seitz, D. N., Serfass, B., Sundqvist, K. M., Tarka, M., Wikus, P., Yellin, S., Yoo, J., Young, B. A., and Zhang, J., "Dark Matter Search Results from the CDMS II Experiment," Science 327, 1619- (Mar. 2010).

[13] Akerib, D. S., Armel-Funkhouser, M. S., Attisha, M. J., Bailey, C. N., Baudis, L., Bauer, D. A., Brink, P. L., Bunker, R., Cabrera, B., Caldwell, D. O., Chang, C. L., Crisler, M. B., Cushman, P., Daal, M., Dixon, R., Dragowsky, M. R., Driscoll, D. D., Duong, L., Ferril, R., Filippini, J., Gaitskell, R. J., Hennings-Yeomans, R., Holmgren, D., Huber, M. E., Kamat, S., Lu, A., Mahapatra, R., Mandic, V., Martinis, J. M., Meunier, P., Mirabolfathi, N., Nelson, H., Nelson, R., Ogburn, R. W., Perera, T. A., Issac, M. C. P., Ramberg, E., Rau, W., Reisetter, A., Ross, R. R., Saab, T., Sadoulet, B., Sander, J., Savage, C., Schnee, R. W., Seitz, D. N., Serfass, B., Sundqvist, K. M., Thompson, J.-P. F., Wang, G., Yellin, S., and Young, B. A., "Exclusion limits on the WIMP-nucleon cross section from the first run of the Cryogenic Dark Matter Search in the Soudan Underground Laboratory," Physical Review D 72, 052009 (Sept. 2005).

[14] Pyle, M., Serfass, B., Brink, P. L., Cabrera, B., Cherry, M., Mirabolfathi, N., Novak, L., Sadoulet, B., Seitz, D., Sundqvist, K. M., Tomada, A., Yen, J. J., and Young, B. A., "Surface Electron Rejection from Ge Detector with Interleaved Charge and Phonon Channels," in [American Institute of Physics Conference Series], Young, B., Cabrera, B., and Miller, A., eds., American Institute of Physics Conference Series 1185, 223-226 (Dec. 2009).

[15] Avignone, III, F. T., Elliott, S. R., and Engel, J., "Double beta decay, Majorana neutrinos, and neutrino mass," Reviews of Modern Physics 80, 481-516 (Apr. 2008).

[16] Freedman, D. Z., "Coherent effects of a weak neutral current," Physical Review D 9, 1389-1392 (Mar. 1974).

[17] Noroozian, O., Day, P. K., Eom, B. H., Leduc, H. G., and Zmuidzinas, J., "Crosstalk Reduction for Superconducting Microwave Resonator Arrays," ArXiv e-prints (June 2012). 